\title{
Predictive risk factors of coronary artery aneury- sms in Kawasaki disease
}

Hyang-Ok Woo, MD, PhD

Department of Pediatrics, Institute of Health Science, Gyeongsang National University College of Medicine, Jinju, Korea

Kawasaki disease (KD) is an acute febrile systemic vasculitis of unknown etiology that occurs during early childhood. Coronary artery lesions (CALs) develop in up to 15\%-25\% of patients with untreated KD. CAL is the most severe complication of KD. Recent studies have reported that epidemiological and clinical characteristics, including the incidence of incomplete cases, coronary artery involvements, and intravenous immunoglobulin (IVIG) nonresponsiveness have changed over time.

CALs develop within 2 weeks of disease onset and are diagnosed via echocardiography. Coronary dilatation was defined as a $z$ score $\geq 2.5$ in $\mathrm{KD}$. The development of coronary aneurysm raises clinical suspicions for the diagnosis of $\mathrm{KD}$. ${ }^{1)}$ For $\mathrm{KD}$ patients with coronary aneurysm, careful planning of follow-up is recommended; in particular, coronary angiography should be routinely performed to appropriately assess patients with large-sized coronary aneurysms. $^{2)}$

KD has no specific diagnostic laboratory markers. Recent studies have investigated factors for predicting resistance to IVIG and CALs. These data include the duration of fever, polymorphonuclear neutrophil (PMN) cell count, hemoglobin, platelet count, C-reactive protein (CRP), transaminase, total bilirubin and N-terminal-pro-brain natriuretic peptide (NTproBNP), albumin, and sodium levels. However, there have been conflicting results, and no single marker has proven to be indicative of KD patients who were resistant to IVIG therapy.

Several previous studies demonstrated that higher PMN percentage, NT-proBNP, total bilirubin, CRP, aspartate aminotransferase, and alanine aminotransferase levels were considered predictive factors for patients with KD resistance to IVIG treatment. Also, lower levels of albumin and sodium were risk factors for no reaction to initial treatment with IVIG. ${ }^{3-5)}$

Accordingly, the serum NT-proBNP levels and PMN percentages are significantly elevated in KD patients with IVIG resistance and CALs and may be useful predictive markers. Above all, the serum level of NT-proBNP was considered to the best single predictive marker for CALs among the biochemical markers of IVIG resistance.

In addition, Ha et al. ${ }^{6}$ showed that IVIG-resistant patients had higher neutrophil-tolymphocyte ratios (NLR) than IVIG-responsive patients in patients with acute febrile phase of $\mathrm{KD}$. Also, KD patients with CALs had lower age-adjusted hemoglobin levels and higher platelet counts than $\mathrm{KD}$ patients without coronary dilatation. These results correlated with the findings of Lee and Song. ${ }^{5)}$

The NLR reflects the balance between inflammation and immune regulation in KD. The high acute ratio in KD patients with a coronary aneurysm suggests a role for neutrophilic inflammation in aneurysm formation. Moreover, the KD patients had normocytic and normochromic red blood cells; thus, anemia shown by low age-adjusted hemoglobin levels may indicate the presence of active inflammation. Platelet counts are not known to increase in proportion to the number of fever days, suggesting that increased platelet counts may reflect a longer period of inflammation. Therefore, higher levels of platelet count and CRP and lower levels of hemoglobin and albumin were found to be risk factors for complications of acute phase
Corresponding author: Hyang-Ok Woo MD, PhD Department of Pediatrics, Institute of Health Science, Gyeongsang National University College of Medicine, 15, Jinju-daero 816 beon-gil, Jinju 52727 , Korea

Tel: +82-55-750-8157

Fax: +82-55-752-9339

E-mail: howoo@gnu.ac.kr

https://orcid.org/0000-0001-8849-9341

Received: 15 January, 2019

Revised: 13 February, 2019

Accepted: 19 February, 2019
Copyright $(\subset 2019$ by The Korean Pediatric Society

This is an open-access article distributed under the terms of the Creative Commons Attribution NonCommercial License (http://creativecommons.org/ licenses/by-nc/4.0/n which permits unrestricted noncommercial use, distribution, and reproduction in any medium, provided the original work is properly cited. 
coronary artery involvement.

Several different risk scores are used to predict IVIG resistance and CALs. Shin et al. ${ }^{7)}$ reported that among the risk scoring systems, the Kobayashi risk score demonstrated significant differences between the IVIG resistance and responder groups in Korean patients with KD. However, Fabi et al. ${ }^{8}$ showed that the Kobayashi, Egami, and Formosa scores were ineffective in predicting IVIG resistance and CALs in a predominantly Caucasian cohort.

In this issue of the Korean Journal of Pediatrics, Jeon et al. ${ }^{9)}$ presented risk factors for the occurrence and persistence of coronary aneurysm in an interesting title in pediatrician's, field of treatment that deals with KD. In this retrospective study, comprising 392 subjects, they reported the risk factors associated with early and late coronary artery outcomes in the patients treated for KD. The subject was selected from the patients who manifested acute phase coronary aneurysm which progressed for more than a year. As a result, the significant factors in predicting coronary aneurysm in the early phase were long duration of fever and high platelet count; and the significant factors for persistence of late coronary aneurysm were initial coronary severity and high white blood count. Although reports on new therapeutic options for initial IVIG-resistant patients, such as steroid, infliximab, or repeated IVIG are available, their effectiveness in reducing the prevalence of coronary aneurysm remains controversial. Jeon et al. ${ }^{9)}$ demonstrated that their findings of a predictive risk factor are comparable to that of Ha et al. ${ }^{6}$, and that Harada scores did not have a significant effect on coronary aneurysm. ${ }^{9)}$ Lin et al. ${ }^{10)}$ suggested that the coronary severity 1 month after $\mathrm{KD}$ onset is most crucial to the late coronary outcomes. Although IVIG use improved the initial severity of coronary lesions, it does not further modify the long-term fate of coronary aneurysms.

In conclusion, the identification of predictive risk factors may distinguish those high-risk patients with coronary artery involvement who might require increased follow-up monitoring and more aggressive treatment. The standard consensus is that Korean KD patients need a highly sensitive and exclusive scoring system.

\section{Conflict of Interest}

No potential conflict of interest relevant to this article was reported.

\section{References}

1. McCrindle BW, Li JS, Minich LL, Colan SD, Atz AM, Takahashi M, et al. Coronary artery involvement in children with Kawasaki disease: risk factors from analysis of serial normalized measurements. Circulation 2007;116:174-9.

2. Bang JS, Kim GB, Kwon BS, Song MK, An HS, Song YW, et al. Longterm prognosis for patients with Kawasaki disease complicated by large coronary aneurysm (diameter $\geq 6 \mathrm{~mm}$ ). Korean Circ J 2017;47: 516-22.

3. Kim MK, Song MS, Kim GB. Factors predicting resistance to intravenous immunoglobulin treatment and coronary artery lesion in patients with Kawasaki disease: analysis of the Korean nationwide multicenter survey from 2012 to 2014. Korean Circ J 2018;48:71-9.

4. Baek JY, Song MS. Meta-analysis of factors predicting resistance to intravenous immunoglobulin treatment in patients with Kawasaki disease. Korean J Pediatr 2016;59:80-90.

5. Lee HY, Song MS. Predictive factors of resistance to intravenous immunoglobulin and coronary artery lesions in Kawasaki disease. Korean J Pediatr 2016;59:477-82.

6. Ha KS, Jang GY, Lee J, Lee KC, Son CS. Laboratory markers in incomplete Kawasaki disease according to coronary artery outcome. Korean Circ J 2018;48:287-95.

7. Shin J, Lee H, Eun L. Verification of current risk scores for Kawasaki disease in Korean children. J Korean Med Sci 2017;32:1991-6.

8. Fabi M, Andreozzi L, Corinaldesi E, Bodnar T, Lami F, Cicero C, et al. Inability of Asian risk scoring systems to predict intravenous immunoglobulin resistance and coronary lesions in Kawasaki disease in an Italian cohort. Eur J Pediatr 2019;178:315-22.

9. Jeon SK, Kim G, Ko H, Byun JH, Lee HD. Risk factors for the occurrence and persistence of coronary aneurysm in Kawasaki disease. Korean J Pediatr 2018 Nov 22 [Epub]. https://doi.org/10.3345/ kjp.2018.07052.

10. Lin MT, Sun LC, Wu ET, Wang JK, Lue HC, Wu MH. Acute and late coronary outcomes in 1073 patients with Kawasaki disease with and without intravenous $\gamma$-immunoglobulin therapy. Arch Dis Child 2015;100:542-7. 